\title{
DUAL EXTREMUM PRINCIPLES RELATING TO COOLING FINS*
}

\author{
BY \\ S. BHARGAVA AND R. J. DUFFIN \\ Carnegie-Mellon University
}

\begin{abstract}
Under consideration is a differential equation $\left(p u^{\prime}\right)^{\prime}=q u$ of the SturmLiouville type where the function $q(x)>0$ is given. The problem is to find a function $p(x)>0$ in $0 \leq x<b$, a constant $b$ and a solution $u(x)$ of the corresponding differential equation such that the energy functional $\int_{0}^{b}\left[p\left(u^{\prime}\right)^{2}+q u^{2}\right] d x$ is maximized when $p(x)$ is subject to the constraint $\int_{0}^{b} p^{\rho} d x \leq K^{\rho}$ and $u$ is subject to the boundary conditions $u=1$ at $x=0$ and $p(d u / d x)=0$ at $x=b$. Here $K>0$ and $\rho \geq 1$ are constants. A key relation $|d u / d x|=\lambda p^{(\rho-1) / 2}$, where $\lambda$ is a positive constant, is found. This criterion leads to explicit solution of the problem. A further consequence of this criterion together with a pair of dual extremum principles is a "duality inequality" giving sharp upper and lower estimates of the maximum value of the energy functional.

This study is a sequel to previous studies on the optimization of cooling fins by Diffin and McLain [1, 2]. In those papers $\rho=1$. However, the latter deals with fins on convex cylinders and thus the Sturm-Liouville equation studied is a partial differential equation.
\end{abstract}

1. Introduction. In [1], Duffin studied the maximization of the functional

$$
\mathfrak{F}=\int_{0}^{b} q(x) u(x) d x
$$

where $q(x)>0$ is a given continuous function in $x \geq 0$ and $u(x)$ is a differentiable function satisfying the Sturm-Liouville differential equation

$$
\frac{d}{d x}\left(p(x) \frac{d u}{d x}\right)=q(x) u \text { in } 0 \leq x \leq b
$$

and satisfying the boundary conditions $u=1$ at $x=0$ and $p(d u / d x)=0$ at $x=b$ and where $p(x)$ is a continuous function subject to the constraints

$$
p(x)>0 \text { in } 0 \leq x<b
$$

and

$$
\int_{0}^{b} p(x) d x=K>0, \quad \text { a given constant. }
$$

In order to find the unknown constant $b>0$ and the unknown functions $p(x)$ and $u(x)$ having the above properties, Duffin eliminated the differential equation and the boundary

* Received February 21, 1972. Prepared under Research Grant DA-ARO-D-31-124-71-G17, Army Research Office (Durham). 
conditions and studied an equivalent maxmax problem. By a variational argument it was shown that the optimal function $u(x)$ should satisfy the condition

$$
\left|\frac{d u}{d x}\right|=\text { constant in } 0 \leq x \leq b .
$$

This criterion led to explicit solution for the unknowns $b, p(x)$, and $u(x)$ maximizing the functional $\mathfrak{H}$.

In this study we have considered the problem of maximizing the functional $\mathfrak{F}(u)$ subjecting $p(x)$ to a more general type of constraint, namely an inequality constraint of the $L_{\rho}$ norm type

$$
\int_{0}^{b} p^{\rho} d x \leq K^{\rho}, \quad \rho \geq 1, \quad K>0
$$

With a view to finding upper and lower estimates for the maximum value $H$, a "duality inequality" is obtained, namely:

$$
\|u\|_{2, \alpha} \geq H^{1 / 2} \geq\|y\|_{2, \beta}^{-1} .
$$

Here \|\|$_{2, \alpha}$ and \|\|$_{2, \beta}$ are certain dual norms and $u(x)$ and $y(x)$ are arbitrary functions except that they satisfy certain smoothness and normalized boundary conditions.

The problem of maximizing the functional $\mathfrak{F C}$ is recast into a minimax or saddle-point problem rather than a maxmax problem. This together with (II) facilitates the formulation of a pair of dual extremum principles for the maximum value $H$, namely

$$
\min _{u}\|u\|_{2, \alpha}=H^{1 / 2}=\max _{y}\|y\|_{2, \beta}^{-1} .
$$

This is proved taking $q(x)$ as a constant and in that case it is shown that the optimal functions $p(x)$ and $u(x)$ satisfy the condition

$$
\left|\frac{d u}{d x}\right|=\lambda(p(x))(p-1) / 2 \text { in } 0 \leq x \leq b
$$

where $\lambda$ is a positive constant. This criterion leads to explicit solutions for the unknowns $b, p(x)$, and $u(x)$ which maximize $\mathfrak{T C}(u)$.

The paper [1] by Duffin mentioned above concerns the optimum design of a cooling fin of limited weight $K$ so as to dissipate the most heat $H$. The thickness, $p(x)$, of the optimum fin is determined by the criterion (I). Previously Schmidt [5] had proposed the criterion (I) but he based his argument on a fallacious physical assumption.

Previous authors, among which Duffin and McLain [2], Wilkins [6, 7], Liu [8, 9], Appl and Hung [10] and Focke [11] are some, have given several developments of the cooling fin problem. But they have not considered the generalization treated here. Moreover, the duality inequality (II) can be suitably employed to obtain upper and lower bounds for the maximum conductance $H$ of the cooling fin. Several ramifications of the duality inequality are given in $[3,4]$.

This study is a sequel to a study on heat transfer in networks [13]. There a network model for the cooling fin was treated. These studies complement each other; the present treatment is more specific and the network treatment is more general. A later study [14] will treat, in an analogous way, a problem in the design of an elastically supported beam for maximum strength. 
2. Statement of the maximization problem and an equivalent formulation. In this section we state a maximization problem and analyze it heuristically to obtain an equivalent minimax problem. Rigorous developments are given in later sections.

Problem 1. To find two functions $u(x)$ and $p(x)$ and a positive number $b$ which have the following properties: $u(x)$ is differentiable in the interval $0 \leq x \leq b$ and $p(x)$ is continuous in the same interval and the two satisfy the Sturm-Liouville differential equation for $u$ :

$$
\frac{d}{d x}\left[p(x) \frac{d u}{d x}\right]=q(x) u(x) \text { in } 0 \leq x \leq b
$$

where $q(x)$ is positive and continuous in $x \geq 0$, and $u$ is to satisfy the boundary conditions

$$
u=1 \text { at } x=0
$$

and

$$
p(x)(d u / d x)=0 \text { at } x=b .
$$

Moreover $p(x)$ has to satisfy the conditions

$$
p(x)>0 \text { in } 0 \leq x<b
$$

and

$$
\int_{0}^{b} p^{\rho} d x \leq K^{\rho}
$$

where $\rho \geq 1$ and $K>0$ are given constants. Under these conditions on $p(x)$ and $u(x)$ we seek to maximize the functional

$$
\mathfrak{H C}(u)=\int_{0}^{b} q(x) u(x) d x
$$

Introducing the functional

$$
E(p, u)=\int_{0}^{b}\left[p u^{\prime 2}+q u^{2}\right] d x, \quad()^{\prime}=d / d x
$$

enables us to recast the problem into a minimax problem. This change is motivated by the following heuristic analysis.

Let $p(x)$ and $u(x)$ be functions satisfying conditions (1)-(4) for some positive number $b$ and be sufficiently smooth in $0 \leq x \leq b$ so that integration by parts below is valid. Let $v$ be an arbitrary smooth function in $0 \leq x \leq b$. Then

$$
E(p, u+v)=E(p, u)+E(p, v)+2 \int_{0}^{b}\left[p u^{\prime} v^{\prime}+q u v\right] d x
$$

Integration by parts gives

$$
\int_{0}^{b}\left[p u^{\prime} v^{\prime}+q u v\right] d x=\int_{0}^{b} v\left[q u-\left(p u^{\prime}\right)^{\prime}\right] d x+\left[\left(p u^{\prime}\right) v\right]_{0}^{b} .
$$

The first integral on the right-hand side vanishes by (1). If we impose $v=0$ at $x=0$ then the boundary term at $x=0$ vanishes and the other boundary term vanishes because of (3). Moreover $u+v$ satisfies the boundary condition (2). Thus (8) becomes 


$$
E(p, u+v)=E(p, u)+E(p, v) \geq E(p, u) .
$$

It follows that if $E(p, \tilde{u})$ is minimized for the class of functions $\tilde{u}$ satisfying (2) but not necessarily (1) then the minimum is achieved by a function $u$ satisfying (1) and (3). This is a standard result of the calculus of variations where (3) is termed a natural boundary condition because it is necessarily satisfied by the minimizing function.

Now, choosing $v=u$ in (9) gives

$$
E(p, u)=-\left[p u^{\prime}\right]_{0} .
$$

On the other hand, choosing $v=1$ in (9) gives

$$
\int_{0}^{b} q u d x=-\left[p u^{\prime}\right]_{0} .
$$

Lemma 1. If the function $u$ satisfies the Euler differential equation (1) corresponding to the saddle functional $E(p, u)$ and if $p$ and $u$ satisfy the boundary conditions (2) and (3) and the condition (4), then

$$
\mathfrak{F C}(u)=E(p, u)
$$

where $\mathfrak{H C}$ and $E$ are given by (6) and (7).

Proof: The lemma is an immediate consequence of (11) and (12).

In view of relations (10) and (13) the following problem is presumably equivalent to Problem 1.

Problem 2. Find

$$
H=\max _{\boldsymbol{D}} \min _{u} E(p, u)
$$

subject to the following conditions: $p \geq 0$ and $p$ has a finite support which is an interval with 0 for the left-hand end point, $p$ is continuous in the closure of its support; the region of integration is the support of $p$, and the integral of $p^{p}$ over the support is not to exceed a given positive constant $K^{p}$; the function $u$ is continuous, has a piecewise continuous first derivative and takes value 1 at $x=0$. Here $q(x)$ occurring in the expression for $E$ is a positive continuous function in $x \geq 0$ and $\rho \geq 1$ is a given constant.

We continue with the heuristic analysis and investigate this problem.

Lemma 2. If $p(x)$ is non-negative and continuous in $0 \leq x \leq b$ and satisfies (5) and $u(x)$ is continuous with piecewise continuous derivative in $0 \leq x \leq b$ then, for $E$ given by (7),

$$
E(p, u) \leq K\left(\int_{0}^{b}\left|u^{\prime}\right|^{\alpha} d x\right)^{2 / \alpha}+\int_{0}^{b} q u^{2} d x,
$$

where the positive constant $\alpha$ is given by

$$
\rho^{-1}+2 \alpha^{-1}=1 \text {. }
$$

There is equality in (15) if

$$
\int_{0}^{b} p^{\rho} d x=K^{\rho}
$$

and

$$
u^{\prime}=-\lambda p^{\rho / \alpha}
$$


where $\lambda$ is a positive constant.

Proof: In

$$
E(p, u)=\int_{0}^{b}\left[p u^{\prime 2}+q u^{2}\right] d x,
$$

applying Hölder's inequality gives

$$
\int_{0}^{b} p u^{\prime 2} d x \leq\left(\int_{0}^{b} p^{\rho} d x\right)^{1 / \rho}\left(\int_{0}^{b}\left|u^{\prime}\right|^{\alpha} d x\right)^{2 / \alpha} \leq K\left(\int_{0}^{b}\left|u^{\prime}\right|^{\alpha} d x\right)^{2 / \alpha},
$$

where $\alpha$ is given by (16). Here (5) has been used. Substituting (19) in (7) gives (15).

If $p$ and $u$ satisfy (17) and (18), there is equality throughout (19) and hence (15) also becomes an equality. This completes the proof of the lemma. We regard the relation (18) as a key result because it enables us to obtain explicit expressions for the solution.

Problem 1a is stated as Problem 1 with $q(x) \equiv q>0$, a constant, and this particular case is our main interest from now on. Similarly, Problem 2a is stated as Problem 2 with $q(x) \equiv q$.

Lemma 3. A pair $b=b_{0}$ and $\lambda=\lambda_{0}$ of positive constants and a solution $u=u_{0}$, $p=p_{0}$ of the system of equations

$$
\begin{gathered}
\frac{d}{d x}\left[p \frac{d u}{d x}\right]=q u(x) \text { in } 0 \leq x \leq b, \\
u^{\prime}=-\lambda p^{\rho / \alpha} \text { in } 0 \leq x \leq b
\end{gathered}
$$

under the boundary conditions

$$
\begin{gathered}
u=1 \text { at } \quad x=0, \\
p(d u / d x)=0 \text { at } x=b
\end{gathered}
$$

and satisfying

$$
\begin{gathered}
p>0 \text { in } 0 \leq x<b, \\
\int_{0}^{b} p^{\rho} d x=K^{\rho}
\end{gathered}
$$

are given by

$$
\begin{gathered}
u_{0}=\left(1-\left(x / b_{0}\right)\right)^{\rho}, \\
p_{0}=\delta_{0}\left(1-\left(x / b_{0}\right)\right)^{2},
\end{gathered}
$$

where

$$
\begin{gathered}
\delta_{0}=q b_{0}^{2} / \rho(\rho+1), \\
b_{d}=\left[\frac{K \rho(\rho+1)(2 \rho+1)^{1 / \rho}}{q}\right]^{\rho /(1+2 \rho)},
\end{gathered}
$$

and

$$
\lambda_{0}=\rho / b_{0} \delta_{0}^{\rho / \alpha} .
$$

Proof: Multiplying (1) by $2 u^{\prime}$ and integrating gives 


$$
-\int_{x}^{b} p^{\prime} u^{\prime 2} d x+p u^{\prime 2}=q u^{2}
$$

Here the boundary condition (3) has been used and an additional condition $u=0$ at $x=b$ has been imposed. Substituting (18) in (25) and integrating gives

$$
\left(\lambda^{2} / \rho\right)(\rho+1) p^{\rho}=q u^{2} .
$$

Here $p(x)$ has been required to satisfy $p=0$ at $x=b$. Eliminating $p(x)$ between (18) and (26) and integrating the result and using the initial condition (2) gives $u(x)$ and then substituting this in (26) gives $p(x)$, namely

$$
u=(1-(x / b))^{\rho}
$$

and

$$
p=\delta(1-(x / b))^{2}
$$

where $\delta, \lambda$ and $b$ satisfy

$$
\begin{gathered}
\frac{1}{b}=\frac{\lambda^{1 / \rho}}{\rho^{(\rho+1) / 2 \rho}}\left(\frac{q}{1+\rho}\right)^{(\rho-1) / 2 \rho}, \\
\delta^{\rho}=\frac{q \rho}{\lambda^{2}(\rho+1)} .
\end{gathered}
$$

Requiring $p$ to satisfy (17) gives

$$
\frac{q \rho b}{\lambda^{2}(\rho+1)(2 \rho+1)}=K^{\rho} .
$$

Eliminating $\lambda$ between (29) and (31) gives $b=b_{0}$, where $b_{0}$ is as in (23). Eliminating $\lambda$ between (29) and (30) and substituting $b=b_{0}$ gives $\delta=\delta_{0}$, where $\delta_{0}$ is as in (22). Now $\lambda=\lambda_{0}$, where $\lambda_{0}$ is as in (24), follows from (22), (23) and (31).

It is contained in Lemma 3 that $p_{0}$ and $u_{0}$ satisfy the differential equation (1) and conditions (2)-(5) for $b=b_{0}$. This was enabled by the relations (17) and (18) of Lemma 2 which render (15) an equality for $p=p_{0}, u=u_{0}$ and $b=b_{0}$. Further it will be seen in Sec. 3 how the inequality (15) can be used to solve the remaining part of Problem 1a. In fact, we show rigorously that $p=p_{0}, u=u_{0}, b=b_{0}$ is the unique solution set solving Problem 1a.

3. Comparison relations and the main proof. In this section we restate Problem 2a relaxing certain conditions and show that $p=p_{0}, u=u_{0}$ of Lemma 3 provide the unique solution of the new problem, called Problem 3 . It is then shown that the solution of Problem 3 is also the unique solution of Problem 2a and finally that $p=p_{0}, u=u_{0}$, $b=b_{0}$ is the unique solution set having the desired property of satisfying (1)-(5) and maximizing $\mathfrak{H C}$ given by (6).

Problem 3. Find

$$
H=\sup _{p} \inf _{v} E(p, u)
$$

subject to the following conditions: $p \geq 0$ and has a finite support which is an interval with 0 for the left-hand end point, $p$ is continuous in the closure of its support; the region of integration is the support of $p$, and the integral of $p^{p}$ over the support is not to exceed 
a given positive constant $K^{\rho}$; the function $u$ is continuous, has piecewise continuous first derivative and takes value 1 at $x=0$. The quantity $q>0$ occurring in the expression for $E$ and $\rho \geq 1$ are given constants.

Lemma 4. Let $p$ be an arbitrary function satisfying the conditions of the p-functions of Problem 3. Let $p_{0}$ and $u_{0}$ be the funclions given by (20)-(23). Then

$$
E\left(p_{0}, u_{0}\right) \geq E\left(p, u_{0}\right) .
$$

Proof: By Lemma 3, for $b=b_{0}$, the functions $p_{0}$ and $u_{0}$ satisfy (17) and (18) of Lemma 2 and (15) becomes an equality for $p=p_{0}, u=u_{0}$ and $b=b_{0}$. Hence

$$
E\left(p_{0}, u_{0}\right)=K\left(\int_{0}^{b_{\bullet}}\left|u_{0}^{\prime}\right|^{\alpha} d x\right)^{2 / \alpha}+\int_{0}^{b_{0}} q u_{0}^{2} d x .
$$

Now let $b$ be the right-hand end point of the support of $p$. It is convenient to consider the two cases $b_{0}<b$ and $b_{0} \geq b$ separately.

Case (i) $b_{0}<b$ : Since $u_{0}\left(b_{0}\right)=0$, by defining $u_{0}$ as zero for $x>b_{0}$ we have a continuous extension of $u_{0}$ and thus the extended function has the admissibility properties of the $u$-functions of Problem 3. So

$$
E\left(p, u_{0}\right)=\int_{0}^{b}\left[p u_{0}^{\prime 2}+q u_{0}^{2}\right] d x \leq K\left(\int_{0}^{b}\left|u_{0}^{\prime}\right|^{\alpha} d x\right)^{2 / \alpha}+\int_{0} q u_{0}^{2} d x
$$

Here we have applied Hölder's inequality

$$
\int_{0}^{b} p u_{0}^{\prime 2} d x \leq\left(\int_{0}^{b} p^{\rho} d x\right)^{1 / \rho}\left(\int_{0}^{b}\left|u_{0}^{\prime}\right|^{\alpha} d x\right)^{2 / \alpha}
$$

and the property of $p$ that $\int_{0}^{b} p^{\rho} d x \leq K^{\rho}$. Now since $u_{0}=0$ in $x>b_{0}$, the right-hand side of the inequality in (35) becomes $E\left(p_{0}, u_{0}\right)$ on using (34), thus giving (33).

Case (ii) $b_{0} \geq b$ : Since the restriction of $u_{0}$ to $0 \leq x \leq b$ satisfies the admissibility properties of the $u$-functions of Problem 3 , we have also in this case

$$
E\left(p, u_{0}\right)=\int_{0}^{b}\left[p u_{0}^{\prime 2}+q u_{0}^{2}\right] d x \leq K\left(\int_{0}^{b}\left|u_{0}^{\prime}\right|^{\alpha} d x\right)^{2 / \alpha}+\int_{0}^{b} q u_{0}^{2} d x
$$

on applying Hölder's inequality as in Case (i) and on using $\left(\int_{0}^{b} p^{\rho} d x\right) \leq K^{\rho}$. Now, in the right-hand side of the inequality of (36)

$$
\int_{0}^{b}\left|u_{0}^{\prime}\right|^{\alpha} d x \leq \int_{0}^{b_{\bullet}}\left|u_{0}^{\prime}\right|^{\alpha} d x
$$

and

$$
\int_{0}^{b} u_{0}^{2} d x \leq \int_{0}^{b} u_{0}^{2} d x
$$

since $b_{0} \geq b$. Using these and (34) in (36) gives (33). This completes the proof of the lemma.

Theorem 1. The pair of functions $\left[p_{0}, u_{0}\right]$ given by (20)-(23) is a solution of Problem 3 .

Proof: Let $p$ be an arbitrary function satisfying the admissibility conditions of the $p$-functions of Problem 3 and $u$ be an arbitrary function satisfying the admissibility conditions of the $u$-functions of Problem 3. Then, since both the restriction of $u_{0}$ to any $0 \leq x \leq b$ with $b \leq b_{0}$ and extension of $u_{0}$ as zero beyond $x=b_{0}$ are admissible, 


$$
\inf _{u} E(p, u) \leq E\left(p, u_{0}\right) \leq E\left(p_{0}, u_{0}\right)
$$

by (33), and therefore

$$
\sup _{p} \inf _{u} E(p, u) \leq E\left(p_{0}, u_{0}\right)
$$

Also,

$$
\sup _{p} \inf _{u} E(p, u) \geq \inf _{u} E\left(p_{0}, u\right)=E\left(p_{0}, u_{0}\right) .
$$

The last equality in (38) is true because $u_{0}$ satisfies the Euler differential equation (1), with $p=p_{0}$, of the positive definite quadratic functional $E\left(p_{0}, u\right)$ and the boundary conditions (2) and (3). The relations (37) and (38) now imply the desired result that

$$
E\left(p_{0}, u_{0}\right)=\sup _{p} \inf _{u} E(p, u) .
$$

The relation (39) is the same as (32) of Problem 3 and shows that $H$ of (32) equals $E\left(p_{0}, u_{0}\right)$.

Theorem 2. The pair of functions $\left[p_{0}, u_{0}\right]$ given by (20)-(23) is the unique solution of Problem 3.

Proof: A proof of uniqueness can be given in essentially the same manner as the uniqueness proof given by Duffin and McLain in Theorem 2 of [2]. We delay giving this proof until Sec. 5 .

Theorem 3. Problem $2 a$ and Problem 3 are equivalent and the pair of functions $\left[p_{0}, u_{0}\right]$ given by (20)-(23) is the only solution of Problem 2a.

Proof: By Theorem 1, the sup inf of (32) of Problem 3 is actually assumed for functions $p=p_{0}$ and $u=u_{0}$ and hence the solution $\left[p_{0}, u_{0}\right]$ of Problem 3 is also a solution of Problem 2a. Here we have also used the fact that the functionals being optimized in the two problems are the same. This shows the equivalence of two problems and that the minimax values of the two problems are equal. Now the solution of Problem 2a is unique. For otherwise it would mean that Problem 3 has another solution besides $\left[p_{0}, u_{0}\right]$. But this contradicts Theorem 2 and the proof is complete.

Theorem 4. Problem 1a is equivalent to Problem 2a and Problem 3 and the pair of functions $\left[p_{0}, u_{0}\right]$ given by (20)-(23) is the unique solution of Problem 1. Summarizing: Problem 1a has the unique solution $p=p_{0}, u=u_{0}, b=b_{0}$ given by

$$
\begin{gathered}
u_{0}=\left(1-\left(x / b_{0}\right)\right)^{\rho}, \\
p_{0}=\delta_{0}\left(1-\left(x / b_{0}\right)\right)^{2},
\end{gathered}
$$

where

$$
\begin{gathered}
\delta_{0}=q b_{0}^{2} / \rho(\rho+1) \\
b_{0}=\left[\frac{K \rho(\rho+1)(2 \rho+1)^{1 / \rho}}{q}\right]^{\rho /(1+2 \rho)}
\end{gathered}
$$

and the maximum value $H$ of the functional

$$
\mathcal{H}(u)=q \int_{0}^{b} u(x) d x
$$




$$
H=\mathfrak{C}\left(u_{0}\right)=E\left(p_{0}, u_{0}\right)=\frac{q b_{0}}{(\rho+1)},
$$

where $b_{0}$ is given by (23) above.

Proof: Since $p_{0}$ is continuous in $0 \leq x \leq b_{0}$ and $u_{0}, u_{0}^{\prime}$ and $\left(p_{0} u_{0}^{\prime}\right)^{\prime}$ are also continuous in $0 \leq x \leq b_{0}$, these conditions are sufficient to justify the integration by parts in (9) for $b=b_{0}, p=p_{0}$ and $u=u_{0}$. Thus the relation (13) with $b=b_{0}, p=p_{0}$ and $u=u_{0}$ shows that the maximum value of Problem 1a cannot exceed the minimax value $H$ of Problem 2a. Here the result of Lemma 3, that the pair of functions $\left[p_{0}, u_{0}\right]$ satisfies the differential equation (1) and the side conditions (2)-(5), with $b=b_{0}$, of Problem 1a, has also been used. By (13) with $b=b_{0}, p=p_{0}$ and $u=u_{0}$ we have

$$
H=\mathfrak{H C}\left(u_{0}\right)=E\left(p_{0}, u_{0}\right) \text {. }
$$

These considerations show that $\left[p_{0}, u_{0}\right]$ is a solution of Problem 1a and the maximum value of Problem 1a equals the minimax value of Problem 2a. The solution of Problem 1a is unique. For otherwise Problem 2a would have another solution apart from $\left[p_{0}, u_{0}\right]$. But this contradicts Theorem 3. To complete the proof of the theorem, it is now enough to see that by (11) with $p=p_{0}, u=u_{0}, b=b_{0}$ relation (41) becomes

$$
H=\mathfrak{H}\left(u_{0}\right)=E\left(p_{0}, u_{0}\right)=-\left[p_{0} u_{0}^{\prime}\right]_{x=0} .
$$

Substituting (20)-(23) in this gives (40).

4. A dual variational approach to Problem 1 and the duality inequality. In this section we give a different approach to the solution of Problem 1 and derive a pair of sharp upper and lower estimates for the maximum value $H$ of Problem 1a. The proof of the following theorem does not depend on the results of the previous sections.

Theorem 5. Let $u(x)$ and $y(x)$ be continuous functions in $0 \leq x \leq b$ with piecewise continuous derivatives there. Let $u$ and $y$ satisfy the boundary conditions

$$
u(0)=1
$$

and

$$
y(0)=1, \quad y(b)=0 .
$$

Let

$$
\left\|u^{\prime}\right\|_{\alpha}=\left(\int_{0}^{b}\left|u^{\prime}\right|^{\alpha} d x\right)^{1 / \alpha} \text { and }\|y\|_{\beta}=\left(\int_{0}^{b}|y|^{\beta} d x\right)^{1 / \beta}
$$

Then define dual norms as

$$
\|u\|_{2, \alpha}=\left[K\left\|u^{\prime}\right\|_{\alpha}^{2}+\int_{0}^{b} q u^{2} d x\right]^{1 / 2}
$$

and

$$
\|y\|_{2, \beta}=\left[K^{-1}\|y\|_{\beta}^{2}+\int_{0}^{b} \frac{y^{\prime 2}}{q} d x\right]^{1 / 2}
$$

where $\alpha$ and $\beta$ are positive constants satisfying 


$$
\alpha^{-1}+\beta^{-1}=1
$$

and $K$ is also a positive constant and $q(x)>0$ is continuous in $0 \leq x \leq b$. Then the following inequality holds:

$$
\|u\|_{2, \alpha} \geq H^{* 1 / 2} \geq\|y\|_{2, \beta}^{-1}
$$

where $H^{*}$ is a constant independent of $u$ and $y$.

Proof: Substituting the boundary conditions (42) and (43) in the identity

$$
[(u y)]_{0}^{b}=\int_{0}^{b}\left(u^{\prime} y+u y^{\prime}\right) d x
$$

gives

$$
-1=\int_{0}^{b}\left(u^{\prime} y+u y^{\prime}\right) d x
$$

and therefore

$$
1 \leq\left|\int_{0}^{b} u^{\prime} y d x\right|+\left|\int_{0}^{b} u y^{\prime} d x\right| .
$$

Here applying Hölder's inequality to the first term of the right-hand side and a weighted Schwarz's inequality to the second term gives

$$
1 \leq\left\|u^{\prime}\right\|_{\alpha}\|y\|_{\beta}+\left(\int_{0}^{b} q u^{2} d x\right)^{1 / 2}\left(\int_{0}^{b} \frac{y^{\prime 2}}{q} d x\right)^{1 / 2} .
$$

Applying Cauchy's inequality to this gives

$$
1 \leq\|u\|_{2, \alpha}\|y\|_{2, \beta} .
$$

The factors on the right-hand side cannot vanish and therefore division gives

$$
\|u\|_{2, \alpha} \geq\|y\|_{2, \beta}^{-1} .
$$

Since the left-hand side is independent of $y$ and the right-hand side is independent of $u$, relation (46) follows for some constant $H^{*}$ independent of $u$ and $y$. This completes the proof of the theorem.

The relation (46) furnishes upper and lower bounds for $H^{*}$ and so we term (46) a "duality inequality". The quantity $H^{*}$ is conceivably non-unique. However, it would seem that under the constraint

$$
\int p^{\rho} d x \leq K^{\rho}, \quad \frac{1}{\rho}+\frac{2}{\alpha}=1, \quad \rho \geq 1,
$$

relation (46) determines a unique $H^{*}$ equal to the maximum value of Problem 1 (with $q(x)$ not a constant). We do not pursue, in this study, either this question or the solution of Problem 1.

However, we show in this section that for $\alpha$ defined by $(1 / \rho)+(2 / \alpha)=1$ and $q(x)=$ constant $=q$ and the usual meaning of $\rho, K$ and $q$-as in Problem $1 \mathrm{a}$-and for $b=\infty$ (in fact, any $b \geq b_{0}, b_{0}$ as in Problem 1a, suffices) (46) determines a unique $H^{*}$ and that $H^{*}=H$ the maximum value of Problem 1a. In such a case, when $H^{*}$ is unique, we say that there is no gap in the duality inequality. 
Theorem 5a. Let $u(x)$ and $y(x)$ be continuous with piecewise continuous first derivatives in $x \geq 0$ so that the integrals in (44a) below are convergent. Let $u$ and $y$ satisfy the boundary conditions

$$
u(0)=1, \quad u \text { bounded as } x \rightarrow \infty
$$

and

$$
y(0)=1, \quad y(x) \rightarrow 0 \text { as } \quad x \rightarrow \infty
$$

Let

$$
\left\|u^{\prime}\right\|_{\alpha}=\left(\int_{0}^{\infty}\left|u^{\prime}\right|^{\alpha} d x\right)^{1 / \alpha}, \quad\|y\|_{\beta}=\left(\int_{0}^{\infty}|y|^{\beta} d x\right)^{1 / \beta} .
$$

Then define dual norms as

$$
\begin{aligned}
& \|u\|_{2, \alpha}=\left[K\left\|u^{\prime}\right\|_{\alpha}^{2}+q \int_{0}^{\infty} u^{2} d x\right]^{1 / 2}, \\
& \|y\|_{2, \beta}=\left[K^{-1}\|y\|_{\beta}^{2}+\frac{1}{q} \int_{0}^{\infty} y^{\prime 2} d x\right]^{1 / 2},
\end{aligned}
$$

where $\alpha$ and $\beta$ are positive constants satisfying

$$
\alpha^{-1}+\beta^{-1}=1, \quad \rho^{-1}+2 \alpha^{-1}=1,
$$

and where $\rho \geq 1, K>0$ and $q>0$ are the constants appearing in Problem 1a (or Problem 3). Then the following duality inequality holds for a constant $H^{*}$ independent of $u$ and $y$ :

$$
\|u\|_{2, \alpha} \geq H^{* 1 / 2} \geq\|y\|_{2, \beta}^{-1} .
$$

Proof: This is an immediate consequence of Theorem 5 on letting $b=\infty$.

Theorem 6. Let $u$ and $y$ satisfy the hypothesis of Theorem 5a and $H$ be the maximum value of Problem 1a. Let $\tilde{u}_{0}$ and $\tilde{y}_{0}$ be defined by

$$
\begin{aligned}
& \tilde{u}_{0}(x)=u_{0}(x) \text { in } 0 \leq x \leq b_{0} \\
& =0 \quad \text { in } x>b_{0} \text {, }
\end{aligned}
$$

and

$$
\begin{aligned}
\tilde{y}_{0}(x) & =-\left(p_{0}(x) u_{0}^{\prime}(x) / H\right) & & \text { in } 0 \leq x \leq b_{0} \\
& =0 & & \text { in } x>b_{0} .
\end{aligned}
$$

Then $\tilde{u}_{0}$ and $\tilde{y}_{0}$ satisfy the admissibility conditions of Theorem $5 \mathrm{a}$ and

$$
\left\|\tilde{u}_{0}\right\|_{2, \alpha}^{2}=H=\left\|\tilde{y}_{0}\right\|_{2, \beta}^{-2}
$$

and hence $H^{*}$ of (46a) is unique and equals $H$.

Proof: Let us define $y_{0}$ by

$$
y_{0}(x)=-\left(p_{0}(x) u_{0}^{\prime}(x) / H\right) \text { in } 0 \leq x \leq b_{0} .
$$

Let us recall for convenience the properties of $p_{0}$ and $u_{0}$. By Lemma 2 and Lemma 3 and (41) of Theorem 4, we have 


$$
H=E\left(p_{0}, u_{0}\right)=K\left(\int_{0}^{b_{\bullet}}\left|u_{0}^{\prime}\right|^{\alpha} d x\right)^{2 / \alpha}+\int_{0}^{b_{\bullet}} q u_{0}^{2} d x
$$

by Lemma 3 and (17) we have

$$
\int_{0}^{b_{\bullet}} p_{0}^{\rho} d x=K^{\rho}
$$

by Lemma 3 and (18) we have

$$
u_{0}^{\prime}=-\lambda_{0} p_{0}^{\rho / \alpha} \text { in } 0 \leq x \leq b_{0} ;
$$

because $u_{0}$ satisfies (1) in $0 \leq x \leq b_{0}$ and $y_{0}$ satisfies (50) we have

$$
y_{0}^{\prime}=-\left(q u_{0} / H\right) \text { in } 0 \leq x \leq b_{0},
$$

and lastly by (50) and (18a)

$$
y_{0}=\frac{\lambda_{0}}{H} p_{0}^{(\rho+1) / 2} \text { in } \quad 0 \leq x \leq b_{0} .
$$

Taking norms in (51) and (52), we have

$$
\begin{gathered}
\int_{0}^{b_{\bullet}} \frac{y_{0}^{\prime 2}}{q} d x=H^{-2} \int_{0}^{b_{\bullet}} q u_{0}^{2} d x \\
\left(\int_{0}^{b_{0}}\left|y_{0}\right|^{\beta} d x\right)^{2 / \beta}=\frac{\lambda_{0}^{2}}{H^{2}}\left(\int_{0}^{b_{0}} p_{0}^{\rho} d x\right)^{2 / \beta}=\frac{\lambda_{0}^{2}}{H^{2}} K^{\rho+1}
\end{gathered}
$$

by (17a) and (45a). On the other hand, $p_{0} u_{0}^{\prime 2}=\lambda_{0}^{2} p_{0}^{\rho}$ so

$$
\begin{aligned}
K\left(\int_{0}^{b_{\bullet}}\left|u_{0}^{\prime}\right|^{\alpha} d x\right)^{2 / \alpha} & =\left(\int_{0}^{b_{\bullet}} p_{0} u_{0}^{\prime 2} d x\right) \text { by } \quad \text { (15a) } \\
& =\lambda_{0}^{2} K^{\rho} \quad \text { by } \quad(17 a) .
\end{aligned}
$$

Then (48), (53), (54) and (55) show that

$$
\begin{aligned}
\left\|\tilde{y}_{0}\right\|_{2, \beta}^{2} & =H^{-2}\left[\int_{0}^{b_{\bullet}} q u_{0}^{2} d x+K\left(\int_{0}^{b_{\bullet}}\left|u_{0}^{\prime}\right|^{\alpha} d x\right)^{2 / \alpha}\right] \\
& =H^{-1}
\end{aligned}
$$

by (15a). Besides, (15a) and (47) show that $\left\|\tilde{u}_{0}\right\|_{2, \alpha}^{2}=H$. The last two equations prove (49). It is clear from the definitions of $\tilde{u}_{0}$ and $\tilde{y}_{0}$ and (49) that $\tilde{u}_{0}$ and $\tilde{y}_{0}$ satisfy the convergence, smoothness and boundary conditions of Theorem $5 \mathrm{a}$. That (46a) determines $H^{*}$ uniquely and $H^{*}=H$ is now seen as follows:

Substituting $u=\tilde{u}_{0}$ and $y=\tilde{y}_{0}$ in (46a) gives

$$
H=\left\|\tilde{u}_{0}\right\|_{2, \alpha}^{2} \geq H^{*} \geq\left\|\tilde{y}_{0}\right\|_{2, \beta}^{-2}=H
$$

and hence $H^{*}=H$. This completes the proof of the theorem.

TheоReм 7. (Dual extremum principles.) Let $u$ and $y$ satisfy the hypothesis of Theorem 5a. Then the following two "DUAL EXTREMUM PRINCIPLES" characterizing the maximum value $H$ of Problem 1a hold:

$$
H=\min _{u}\left[K\left(\int_{0}^{\infty}\left|u^{\prime}\right|^{\alpha} d x\right)^{2 / \alpha}+\int_{0}^{\infty} q u^{2} d x\right]
$$


and

$$
H^{-1}=\min _{\nu}\left[K^{-1}\left(\int_{0}^{\infty}|y|^{\beta} d x\right)^{2 / \beta}+\int_{0}^{\infty} \frac{y^{\prime 2}}{q} d x\right] .
$$

Proof: This is a direct consequence of Theorem 6 and Theorem 5a, in particular (46a) and (49).

Corollary 1. Theorem 7 shows that (46a) with $H^{*}$ replaced by $H$, can be used to give sharp estimates of $H$ the maximum value of Problem 1a.

5. Uniqueness proof. In this section we prove a statement made in Section 3.

Theorem 2. The pair of functions $\left[p_{0}, u_{0}\right]$ given by $(20)-(23)$ is the unique solution of Problem 3.

Proof: Assume that $P(x)$ is a function satisfying the admissibility conditions of the $p$-functions of Problem 3 and that $U(x)$ satisfies the admissibility conditions of the $u$-functions such that $E(P, u)$ is minimized by $U$ and

$$
E(P, U)=E\left(p_{0}, u_{0}\right)
$$

Then

$$
E\left(P, u_{0}\right) \geq E(P, U)
$$

But by Lemma 4

$$
E\left(p_{0}, u_{0}\right) \geq E\left(P, u_{0}\right) .
$$

Now (56), (57) and (58) imply that (57) is actually an equality. Thus

$$
E\left(P, u_{0}\right)=E(P, U)=E\left(p_{0}, u_{0}\right) .
$$

Considering $E(P, u)$ as a quadratic functional in $u$, by the parallelogram law for quadratic functionals we have

$$
0 \leq E\left(P, U-u_{0}\right)=2 E(P, U)+2 E\left(P, u_{0}\right)-4 E(P, Z)
$$

where $Z=\left(U+u_{0}\right) / 2$. Because $Z$ satisfies the boundary condition $Z=1$ at $u=0$, $E(P, Z) \geq E(P, U)$. Now this and (59) and (60) imply that $E\left(P, U-u_{0}\right)=0$ and hence

$$
U \equiv u_{0} \text { in } \quad 0 \leq x \leq b,
$$

where $b$ is the right-hand end point of support of $P$. This also shows that

$$
E\left(P, u_{0}\right)=\min _{u} E(P, u)
$$

since the left-hand side equals $E(P, U)$ and $E(P, U)=\min _{u} E(P, u)$. Now, it remains to show that $P \equiv p_{0}$ and $b=b_{0}$.

Since $P$ is continuous and $u_{0}$ is continuous with piecewise continuous derivative in $0 \leq x \leq b$, and $u_{0}$ has the minimizing property (62) in the class of continuous functions with piecewise continuous first derivatives in $0 \leq x \leq b$ and satisfying the boundary condition $u(0)=1$, we may apply the "second lemma" of the calculus of variations [12]. The details will be omitted but it results that $P$ satisfies

$$
P u_{0}^{\prime}=\text { constant }+\int_{0}^{x} q u_{0} d x \text { in } 0 \leq x \leq b,
$$


and the natural boundary condition

$$
P u_{0}^{\prime}=0 \text { at } x=b .
$$

Integrating $E\left(P, u_{0}\right)$ by parts and using (63) and (64) gives

$$
E\left(P, u_{0}\right)=-\left[P u_{0}^{\prime}\right]_{x=0} .
$$

Similarly,

$$
E\left(p_{0}, u_{0}\right)=-\left[p_{0} u_{0}^{\prime}\right]_{x=0} .
$$

It follows from (59), (65) and (66) that

$$
P=p_{0}=\delta_{0} \text { at } x=0 .
$$

Here we have used (20) and (21) and $\delta_{0}$ is given by (22). Now, on using the expression for $u_{0}$ given by (20) and differentiating, (63) gives a first-order linear equation of the Cauchy-Euler type in $D$, namely

$$
\left(1-\frac{x}{b_{0}}\right) P^{\prime}-\frac{(\rho-1)}{b_{0}} P=-\frac{q b_{0}}{\rho}\left(1-\frac{x}{b_{0}}\right)^{2}
$$

where

$$
D=(\text { support of } P) \cap \text { (support of } p_{0} \text { ). }
$$

Let $B$ be the right-hand end point of the support of $D$. Then $B=\min \left[b, b_{0}\right]$. Now as $p_{0}$ satisfies (68) and $P$ and $p_{0}$ satisfy the same initial condition (67), it follows that

$$
P \equiv p_{0} \quad \text { in } D .
$$

So it is enough to show $b=b_{0}$. Suppose that $b<b_{0}$. Then

$$
\begin{aligned}
E\left(P, u_{0}\right)= & \int_{0}^{b}\left[P u_{0}^{\prime 2}+q u_{0}^{2}\right] d x \\
= & \int_{0}^{b}\left[p_{0} u_{0}^{\prime 2}+q u_{0}^{2}\right] d x \\
& <\int_{0}^{b_{0}}\left[p_{0} u_{0}^{\prime 2}+q u_{0}^{2}\right] d x=E\left(p_{0}, u_{0}\right) .
\end{aligned}
$$

Here (70) and the fact $p_{0} \not \equiv 0 \not \equiv u_{0}$ in $b \leq x \leq b_{0}$ are used. The inequality (71) contradicts (.59) and hence $b>b_{0}$ is the only other possibility if not $b=b_{0}$. So, if $b>b_{0}$,

$$
K^{\rho} \geq \int_{0}^{b} P^{\rho} d x=\left(\int_{0}^{b_{\bullet}} P^{\rho} d x\right)+\left(\int_{b_{\bullet}}^{b} P^{\rho} d x\right)>K^{\rho} .
$$

Here $\int_{0}^{b_{0}} p_{0}^{\rho} d x=K^{\rho},(70)$ and also the fact $P \not \equiv 0$ in $b_{0} \leq x \leq b$ are used. But (72) is absurd and thus $b>b_{0}$ is untenable and $b=b_{0}$. Thus the proof of the theorem is complete.

\section{REFERENCES}

[1] R. J. Duffin, A variational problem relating to cooling fins, Jour. Math. and Mech. 8, 47-56 (1959)

[2] R. J. Duffin and D. K. McLain, Optimum shape of a cooling fin on a convex cylinder, Jour. Math. and Mech. 17, 769-784 (1968) 
[3] R. J. Duffin, Duality inequalities of mathematics and science, in Nonlinear programming, edited by J. B. Rosen, O. L. Mangasarian, and K. Ritter, Academic Press Inc., New York, 401-423, (1970)

[4] R. J. Duffin, Network models, in Proceedings of the Symposium on Mathematical Aspects of Electrical Network Theory, SIAM-AMS Proceedings 3, 65-91 (1969)

[5] E. Schmidt, Die Wärmeübertragung durch Rippen, Zeit. d. ver. deutch Ing. 70, 885-890 (1926)

[6] J. E. Wilkins, Jr., Minimum mass thin fins for space radiators, in Proc. Heat Transfer and Fluid Mech. Inst., Stanford University Press, 1960, 229-243

[7] J. E. Wilkins, Jr., Minimum mass thin fins and constant temperature gradients, J. Soc. Ind. Appl. Math. 10, 62-73 (1962)

[8] C. Y. Liu, A variational problem with applications to cooling fins, J. Soc. Ind. Appl. Math. 10, 19-29 (1962)

[9] C. Y. Liu, A variational problem relating to cooling fins with heat generation, Quart. Appl. Math. 19, 245-251 (1962)

[10] F. C. Appl and H. M. Hung, A principle for convergent upper and lower bounds, Int. J. Mech. Sci. 6, 381-389 (1964)

[11] R. Focke, Die Nadel als Kühlelement, Forschung auf dem Gebiete des Ingenieurwesens 13, 34-42 (1942)

[12] N. I. Akhiezer, The calculus of variations (translated from the Russian), Blaisdell Pub. Co., New York (1962), 106-107

[13] S. Bhargava and R. J. Duffin, Network models for maximization of heat transfer under weight constraints, Networks 2, 285-299 (1972)

[14] S. Bhargava and R. J. Duffin, Dual extremum principles relating to optimum beam design, Arch. Rat. Mech. Anal. (1973), to appear 